\title{
Aclaramiento del déficit de base estándar como pronóstico de mortalidad en choque séptico**
}

Standard base deficit clearance as prognostic factor of mortality in septic shock Depuração do déficit de base padrão como prognóstico de morte no choque séptico Gabriel García Gómez, ${ }^{\star}$ Jesús Salvador Sánchez Díaz, ${ }^{\star}$ Karla Gabriela Peniche Moguel, ${ }^{\star}$ Enrique Monares Zepeda, ${ }^{\ddagger}$
Enrique Antonio Martínez Rodríguez, ${ }^{\S}$ María Verónica Calyeca Sánchez ${ }^{\star}$

\section{RESUMEN}

Introducción: A finales de la década de los cincuenta, el concepto de exceso de base (EB) fue introducido por Siggaard-Andersen como marcador sérico de los problemas estrictamente metabólicos. Hoy en día, la base (B) ha sido estandarizada con resultados más efectivos cuando utilizamos la base estándar (Becf) en la evaluación clínica. El lactato y la base estándar medidos al ingresar a la Unidad de Cuidados Intensivos (UCI) son de utilidad pronóstica en los pacientes críticamente enfermos. Decidimos medir el aclaramiento del déficit de base estándar en los pacientes con choque séptico en las 24 horas subsecuentes a partir del ingreso y evaluar su utilidad pronóstica para la mortalidad. Material y métodos: Estudio de cohorte, retrospectivo, descriptivo y analítico, que incluyó pacientes con diagnóstico de choque séptico según el tercer Consenso Internacional de Sepsis y Choque Séptico (sepsis-3) ingresados a la Unidad de Cuidados Intensivos (UCl) durante el periodo comprendido de mayo de 2015 a mayo de 2019 . Los pacientes se asignaron al grupo $<11 \%$ o al grupo $\geq 11 \%$ según su porcentaje (\%) de aclaramiento de déficit de base estándar a las 24 horas. Resultados: Se incluyeron 118 pacientes que cumplieron con los criterios de inclusión. Del total, 43 pacientes se asignaron al grupo de aclaramiento de déficit de base estándar $<11 \%$ y 75 pacientes al grupo de aclaramiento de déficit de base estándar $\geq 11 \%$. En el grupo con aclaramiento $<11 \%$, la mortalidad fue de $65.1 \%$ y de $36 \%$ para el grupo con aclaramiento $\geq 11 \%$ con $p=<0.05$. Conclusión: El aclaramiento del déficit de base estándar $<11 \%$ a las 24 horas de ingreso a la Unidad de Cuidados Intensivos incrementa el riesgo de muerte en el choque séptico. El porcentaje de aclaramiento del déficit de base estándar es una excelente alternativa, pues su desempeño para predecir la mortalidad tiene una capacidad pronóstica similar a la del lactato.

Palabras clave: Choque séptico, aclaramiento del déficit de base estándar, mortalidad.

\section{ABSTRACT}

Introduction: The concept of base excess (BE) was introduced by SiggaardAndersen in the late 1950s as a serum marker of strictly metabolic problems. Today the base $(B)$ has been standardized with more effective results when we use the standard base (ecfB) in the clinical evaluation. Lactate and the standard base measured upon admission to the Intensive Care Unit (ICU) are of prognostic utility in critically ill patients. We decided to measure the standard base deficit clearance at 24 hours after admission in patients with septic shock and evaluate its prognostic utility for mortality.

Material and methods: Cohort, retrospective, descriptive, analytical study that included patients with diagnosis of septic shock according to the third international consensus of sepsis and septic shock (sepsis-3) admitted to the ICU in the period from May 2015 to May 2019. Patients were assigned to belong to group $<11 \%$ or group $\geq 11 \%$ according to their percentage (\%) of standard base deficit clearance at 24 hours.

Results: 118 patients who met the inclusion criteria were included. Of the total, 43 patients were assigned to the standard base deficit clearance group $<11 \%$ and 75 patients to the standard base deficit clearance group $\geq 11 \%$. In the group with clearance $<11 \%$ the mortality was 65.1 and $36 \%$ for the group with clearance $\geq 11 \%$ with $p=<0.05$.

Conclusion: Clearance the standard base deficit $<11 \%$ after 24 hours of admission to the ICU increases the risk of death in septic shock. The percentage of standard base deficit clearance is an excellent alternative, since its performance to predict mortality has a prognostic capacity similar to that of lactate.

Keywords: Septic shock, standard base deficit clearance, mortality.

** Ganador del Premio Académico «Dr. Mario Shapiro» 2019. Primer lugar.

* Hospital de Especialidades Núm. 14, Centro Médico Nacional «Adolfo Ruiz Cortines», Instituto Mexicano del Seguro Social. Veracruz, Veracruz.

₹ Hospital San Ángel Inn Universidad. Ciudad de México, México.

$\S$ Centro Médico ABC. Ciudad de México, México.

Recepción: 30/08/2019. Aceptación: 23/10/2019.

Este artículo puede ser consultado en versión completa en www.medigraphic.com/medicinacritica

\section{RESUMO}

Introdução: O conceito de excesso de base (EB) foi introduzido por SiggaardAndersen no final da década de 1950 como um marcador sérico de problemas estritamente metabólicos. Hoje a base (B) foi padronizada com resultados mais eficazes quando usamos a base padrão (Becf) na avaliação clínica. O lactato e a base padrão medidas na admissão na unidade de terapia intensiva (UTI) são de utilidade prognóstica em pacientes críticos. Decidimos medir a depuração do déficit de base padrão 24 horas após a internação em pacientes com choque séptico e avaliar sua utilidade prognóstica para a mortalidade.

Material e métodos: Estudo de coorte, retrospectivo, descritivo, analítico que incluiu pacientes com diagnóstico de choque séptico de acordo com o terceiro consenso internacional de sepse e choque séptico (sepse-3) admitidos na UTI no período de maio de 2015 a maio de 2019. Os pacientes foram designados para pertencer ao grupo $<11 \%$ ou grupo $\geq 11 \%$, de acordo com a porcentagem (\%) de depuração do déficit de base às 24 horas.

Resultados: Foram incluídos 118 pacientes que atenderam aos critérios de inclusão. Do total, 43 pacientes foram designados para o grupo de depuração do déficit de base padrão $<11 \%$ e 75 pacientes para o grupo de depuração do déficit de base padrão $\geq 11 \%$. No grupo com depuração $<11 \%$ a mortalidade foi de $65.1 \%$ e $36 \%$ no grupo com depuração $\geq 11 \%$ com $p=<0.05$.

Conclusão: A depuração do déficit de base padrão < 11\% após 24 horas de internação na UTI aumenta o risco de morte em choque séptico. A porcentagem de depuração do déficit de base padrão é uma excelente alternativa, uma vez que seu desempenho na predição da mortalidade possui capacidade prognóstica semelhante à do lactato.

Palavras-chave: Choque séptico, depuração do déficit de base padrão, mortalidade.

\section{INTRODUCCIÓN}

A pesar de todo el cúmulo de conocimientos sobre el choque séptico y la disminución del tiempo para cumplir tempranamente los bundles de la campaña «Sobreviviendo a la sepsis», todavía el choque séptico alcanza una mortalidad que varía de 18 al $35 \%$. A nivel mundial, 14 millones de pacientes sobrevivirán al choque séptico, pero la gran mayoría presentará incapacidad a largo plazo y deterioro de la calidad de vida por la morbilidad. Sólo la mitad de los pacientes que padecieron choque séptico tendrá una recuperación completa. ${ }^{1,2}$ El tratamiento del choque séptico tiene enormes implicaciones financieras, de hecho, en Estados Unidos, la sepsis ocupa el primer lugar en gastos de hospitalización. En México, la mortalidad del choque séptico es alrededor de $30.4 \%$ y su etiología abdominal es la más frecuente con cerca de la mitad de los casos, seguida de la pulmonar con un tercio. ${ }^{3}$ El costo de un día de estancia en la Unidad de Cuidados Intensivos ( $\mathrm{UCl}$ ) es de alrededor de 34,232 pesos mexicanos, ${ }^{4}$ y si tomamos en cuenta que la principal causa de choque es la sepsis, ${ }^{5}$ este dato se ve alarmante, considerando que la tendencia de este serio problema de salud está a la alza. ${ }^{6}$

A finales de la década de los cincuenta, el concepto de exceso de base (EB) fue introducido por Siggaard-An- 
dersen como marcador sérico de problemas estrictamente metabólicos. Hoy en día, la base (B) ha sido estandarizada con resultados más efectivos cuando utilizamos la base estándar (Becf) en la evaluación clínica. ${ }^{7}$

La escuela de Boston (a favor del uso de bicarbonato) y la de Copenhague (a favor del uso de exceso de base estándar) no «coinciden» en cuál es la mejor herramienta para valorar las alteraciones metabólicas de los problemas ácido-base. ${ }^{8}$ Lo cierto es que Peter Stewart consideró al bicarbonato $\left(\mathrm{HCO}_{3}^{-}\right)$como una variable dependiente y demasiado simple para explicar todos estos problemas. ${ }^{9}$ En este contexto, existen diferentes sistemas amortiguadores, el más conocido pero no el único, es el $\mathrm{HCO}_{3}^{-}$, por lo que Stewart argumentó que variables como el agua, cloro, fosfato, albumina, lactato y aniones no medidos pueden ser la explicación de las alteraciones metabólicas. ${ }^{10}$ Entonces, la base es la cantidad de $\mathrm{mEq}$ (ácidos o bases) que se necesitan para corregir una alteración metabólica y «normalizar» el $\mathrm{pH}$. Una base más negativa (déficit de base) sugiere acidosis metabólica y una base más positiva (exceso de base) sugiere alcalosis metabólica. ${ }^{11}$

Desde 1980 se clasifica a los pacientes críticamente enfermos según su gravedad, con el fin de comparar y evaluar sus resultados, los cuales, en la UCl, dependerán de diversos factores presentes desde el día de su ingreso. Las escalas de gravedad se componen de dos partes: la primera, puntuación en sí misma (a mayor número, mayor gravedad) y la segunda, modelo de probabilidad (ecuación probabilística). ${ }^{12}$ El lactato y la base estándar medidos al ingreso a la $\mathrm{UCI}$ son de utilidad pronóstica en los pacientes críticamente enfermos, pues sus niveles séricos predicen mortalidad a través de la puntuación en sí misma. ${ }^{13}$ Pero la importancia real va más allá de un valor absoluto, pues es mejor medir su aclaramiento a través de un tiempo determinado. ${ }^{14}$

Desde hace 35 años, Vincent y colaboradores ${ }^{15}$ hablaron del aclaramiento de lactato en los pacientes en estado de choque. La disminución mayor a $5 \%$ en la primera hora de tratamiento se asoció con mejores resultados, aunque algunos no están de acuerdo con el concepto "aclaramiento", pues la cantidad de lactato en sangre, como de cualquier otra sustancia es dependiente del balance entre la producción y la eliminación. ${ }^{16}$

Una revisión sistemática en diferentes poblaciones de pacientes ingresados a la UCI documentó la utilidad de la medición de lactato para predecir resultados. Se concluyó que el aclaramiento del lactato a lo largo del tiempo se asocia con una menor mortalidad. ${ }^{17}$ Independientemente de si es por disminución en la producción o por incremento en la eliminación, un sello distintivo de malos resultados en el estado de choque es mantener los niveles séricos elevados de lactato o falta de aclaramiento. ${ }^{18,19}$ Por un lado, los estudios han documentado la superioridad del déficit de base estándar con respecto al lactato para predecir la mortalidad en la UCI. ${ }^{20}$ Por otro lado, su correlación al ingreso $(r=0.32)$ y a las 24 horas $(r=0.55)$ en pacientes con trauma, pancreatitis y cirugía abdominal mayor no es buena. ${ }^{21}$ Sin embargo, algunos autores han documentado que los valores de déficit de base estándar de $-4 \mathrm{mEq} / \mathrm{L}$ predicen hiperlactatemia en los pacientes con sepsis de por lo menos 3.5 $\mathrm{mEq} / \mathrm{L}$ con una sensibilidad de $91.1 \%$ y especificidad de $88.4 \%{ }^{22}$

No está en duda la utilidad pronóstica del lactato en los pacientes con choque séptico; lo cierto es que su disponibilidad en nuestro medio es menos frecuente que el déficit de base estándar. De esta manera, el déficit de base estándar se convierte en una excelente alternativa. Decidimos medir el aclaramiento del déficit de base estándar a las 24 horas a partir del ingreso en los pacientes con choque séptico y evaluar su utilidad pronóstica para mortalidad.

Justificación: Actualmente el choque séptico se considera un serio problema de salud con enormes implicaciones financieras, de hecho, es la principal causa de ingreso a las unidades de cuidados intensivos polivalentes. Clasificar a los pacientes según su gravedad es útil para comparar y evaluar los resultados. El lactato es una variable ampliamente estudiada, pero con poca disponibilidad en nuestro medio, por lo que el déficit de base estándar se convierte en una excelente alternativa, además de existir buena correlación entre ellas, por lo que el déficit de base podría utilizarse como sustituto y agregarse en nuestros objetivos al momento de reanimar en esta población de pacientes.

Planteamiento del problema: ¿Es útil el aclaramiento del déficit de base estándar medido a las 24 horas como pronóstico de mortalidad en choque séptico?

Objetivo: Determinar la utilidad del aclaramiento del déficit de base estándar medido a las 24 horas como pronóstico de mortalidad en choque séptico.

\section{MATERIAL Y MÉTODOS}

Se autorizó por el Comité de Investigación y Ética del hospital. Estudio de riesgo mínimo de no intervención.

Tipo de estudio: Estudio de cohorte, retrospectivo, descriptivo y analítico.

Universo de trabajo y lugar de desarrollo: Pacientes con diagnóstico de choque séptico según el tercer Consenso Internacional de Sepsis y Choque Séptico (sepsis-3) ingresados en la Unidad de Cuidados Intensivos en el periodo comprendido entre mayo de 2015 a mayo de 2019.

Criterios de inclusión: Pacientes con diagnóstico de choque séptico según el tercer Consenso Internacional de Sepsis y Choque Séptico (sepsis-3) ingresados en la Unidad de Cuidados Intensivos. Pacientes con 
gasometría arterial al ingreso y a las 24 horas de estancia en la Unidad de Cuidados Intensivos. Pacientes mayores de 18 años.

Criterios de exclusión: Pacientes con registro de variables incompleto. Pacientes menores de 18 años.

Criterios de eliminación: Pacientes que concluyan su tratamiento médico en otra terapia intensiva. Paciente embarazada. Paciente con orden de no reanimación o de máximo alcance terapéutico.

Procedimiento: Los pacientes que cumplieron con los criterios de inclusión se asignaron al grupo $<11 \%$ o al grupo $\geq 11 \%$, según su porcentaje (\%) de aclaramiento de déficit de base estándar a las 24 horas, el cual se obtuvo de la gasometría arterial con la siguiente fórmula: (déficit de base estándar de ingreso - déficit de base estándar a las 24 horas)/déficit de base estándar de ingreso * 100 . Se realizó el registro de variables en la hoja de recolección de datos. Además, se consideró al paciente como sobreviviente al ser dado de alta de la UCl o al llegar al día 30 de estancia.

Análisis estadístico: Para el análisis estadístico se inició la prueba de Shapiro-Wilk con el fin de determinar el tipo de distribución de las variables cuantitativas, ya sea normal o no normal, presentándolas como media (desviación estándar) o mediana (rango intercuartilar) respectivamente. Las variables cualitativas se expresan como frecuencia (porcentaje). Posteriormente se construyó una curva Receiver Operating Characteristic (ROC) evaluando el desempeño del aclaramiento del déficit de base estándar y del lactato en la detección de mortalidad. Se obtuvieron los valores de área bajo la curva $A B C$ con intervalo de confianza al 95\% (IC 95\%) y el valor de p, para posteriormente seleccionar un punto corte y dividir la muestra con base en dicho punto. El mejor punto de corte determinado para el aclaramiento del déficit de base estándar fue de $11 \%$ y de $10 \%$ para lactato. Se compararon las $A B C$ de déficit de base estándar y de lactato mediante la prueba de Hanley McNeil. Los subgrupos obtenidos se compararon utilizando la prueba de $t$ de Student o $U$ de Mann Whitney según la distribución de las variables cuantitativas, normal y no normal respectivamente. Las variables cualitativas se compararon mediante la prueba de $\chi^{2}$ o exacta de Fisher según corresponda. Se realizó un modelo de regresión logística bivariado con la variable de mortalidad como dependiente y con aquellas variables con diferencia significativa en las comparaciones previas como variables independientes, presentando el modelo con los valores de B, p, Exp (B) con IC 95\%. La estimación de la función de supervivencia se realizó con el método de Kaplan-Meier. Se realizó un modelo de regresión logística de Cox. Se consideró significativa toda $p<0.05$. El análisis estadístico se realizó con el programa SPSS TM 22.

\section{RESULTADOS}

En el periodo considerado se incluyeron 118 pacientes, los cuales cumplieron con los criterios de inclusión. Del total, 43 pacientes se asignaron al grupo de aclaramiento de déficit de base estándar $<11 \%$ y 75 pacientes al grupo de aclaramiento de déficit de base estándar $\geq$ $11 \%$. En el grupo con aclaramiento $<11 \%, 62.8 \%$ fue de mujeres y en el grupo con aclaramiento $\geq 11 \%$, fue de $50.7 \%$. La edad tuvo una mediana de 65 y 53 años con relevancia estadística, pero las variables talla y peso no tuvieron relevancia estadística.

La media de SAPS II fue de 72.79 y 60.21 con $p=$ $<0.05$ para el grupo de aclaramiento $<11 \%$ y $\geq 11 \%$, respectivamente. La comorbilidad más frecuente fue la hipertensión arterial sistémica (HAS) seguida de la diabetes mellitus tipo 2 (DM2) sin diferencia estadística. La etiología abdominal fue la más frecuente en ambos grupos. En las variables como el índice de choque modificado y el $\Delta \mathrm{p}(\mathrm{v}-\mathrm{a}) \mathrm{CO}_{2}$ no hubo diferencia estadísticamente significativa. $\mathrm{La} \mathrm{SvcO}_{2}$ y la norepinefrina fueron diferentes en ambos grupos con diferencia estadística; la mediana fue de $64 \%$ y $72 \%$ y $0.70 \mu \mathrm{g} / \mathrm{kg} /$ min y $0.21 \mu \mathrm{g} / \mathrm{kg} / \mathrm{min}$ respectivamente para el grupo de aclaramiento $<11 \% \mathrm{y} \geq 11 \%$.

No existió diferencia significativa en las variables creatinina, urea y balance de líquidos, pero sí en la uresis. Las variables $\mathrm{pH}, \mathrm{HCO}_{3}{ }^{-}$, base, lactato y GIF reflejaron mayor severidad de la acidosis metabólica en el grupo de aclaramiento $<11 \%$ con diferencia estadísticamente significativa; esta diferencia no se presentó en la DIFa, DIFe y $\mathrm{PaCO}_{2}$. Los días de ventilación mecánica fueron menos en el grupo de aclaramiento < 11\% (3 días) respecto del grupo $\geq 11 \%$ (4 días) con diferencia estadística, lo mismo para los días de estancia en $\mathrm{UCl}$ con tres y cinco días para el grupo de aclaramiento < $11 \%$ y $\geq 11 \%$ respectivamente. La necesidad de terapia de reemplazo renal lenta continua (TRRLC) fue la misma para ambos grupos. En el grupo con aclaramiento < $11 \%$ la mortalidad fue de $65.1 \%$ y de $36 \%$ para el grupo con aclaramiento $\geq 11 \%$ con $p=<0.05$ (Tabla 1).

Las Figuras $1 A$ y $1 B$ muestran la curva ROC del porcentaje (\%) de aclaramiento del déficit de base estándar y del lactato respectivamente para mortalidad. En la Tabla 2 se puede observar el ABC (0.68) del porcentaje de aclaramiento del déficit de base con $11 \%$ como mejor punto de corte, con sensibilidad de $72 \%$ y especificidad de $51 \%$ con $p=0.00$; también el ABC (0.72) del porcentaje de aclaramiento del lactato con $10 \%$ como mejor punto de corte, con sensibilidad de $72 \%$ y especificidad de $71 \%$ con $p=0.00$. Se compararon las ABC mediante la prueba de Hanley McNeil sin encontrar diferencia significativa $(p>0.05)$ en el desempeño de los puntos de corte seleccionados para mortalidad. En las Figuras $2 A$ y $2 B$ se muestra la estimación de la función de su- 
pervivencia mediante el método de Kaplan-Meier de las variables del porcentaje de aclaramiento del déficit de base estándar y del lactato. Se realizó un modelo de regresión logística de Cox obteniendo un log-rank $<0.05$ para ambas variables.

\section{DISCUSIÓN}

Podemos determinar el grado o la severidad de la acidosis metabólica con el déficit de base estándar, el cual resulta más confiable que el $\mathrm{HCO}_{3}{ }^{-}$, debido a que este último pudiera estar influenciado por la $\mathrm{pCO}_{2}$ (presión de dióxi-

Tabla 1: Análisis bivariado según el porcentaje (\%) de aclaramiento del déficit de base estándar.

\begin{tabular}{|c|c|c|}
\hline Variable & $<11 \%(n=43)$ & $\geq 11 \%(n=75)$ \\
\hline Género femenino (\%) & 62.8 & 50.7 \\
\hline Edad, años (RI) & $65(51-72)^{*}$ & $53(42-63)^{*}$ \\
\hline Talla, cm (DE) & $163.95( \pm 8.60)$ & $163.63( \pm 8.78)$ \\
\hline Peso, kg (RI) & $70(60-80)$ & $73(63-90)$ \\
\hline SAPS II, puntos (DE) & $72.79( \pm 12.75)^{*}$ & $60.21( \pm 11.76)^{*}$ \\
\hline \multicolumn{3}{|l|}{ Comorbilidades } \\
\hline Diabetes (\%) & 48.8 & 42.7 \\
\hline Hipertensión (\%) & 60.5 & 42.7 \\
\hline Enfermedad renal crónica (\%) & 20.9 & 18.7 \\
\hline Cardiopatías (\%) & 16.3 & 6.7 \\
\hline \multicolumn{3}{|l|}{ Origen de la infección } \\
\hline Pulmonar (\%) & 23.3 & 30.7 \\
\hline Abdominal (\%) & 53.5 & 44.0 \\
\hline Urinaria (\%) & 16.3 & 18.7 \\
\hline Tejido blando (\%) & 2.3 & 2.7 \\
\hline Índice de choque modificado (DE) & $1.46( \pm 0.59)$ & $1.12( \pm 0.56)$ \\
\hline $\mathrm{SvCO}_{2}(\%)(\mathrm{RI})$ & $64(57-75)^{*}$ & $72(66-77)^{\star}$ \\
\hline$\Delta \mathrm{p}(\mathrm{v}-\mathrm{a}) \mathrm{CO}_{2} \mathrm{mmHg} / \mathrm{mL}(\mathrm{DE})$ & $6.38( \pm 3.54)$ & $4.62( \pm 2.68)$ \\
\hline Norepinefrina $\mu \mathrm{g} / \mathrm{kg} / \mathrm{min}(\mathrm{RI})$ & $0.70(0.21-0.97)^{\star}$ & $0.21(0.10-0.31)^{\star}$ \\
\hline Creatinina mg/dL (RI) & $2.70(1.70-4.50)$ & $2.20(1.10-3.50)$ \\
\hline Urea mg/dL (RI) & $105.00(65.00-145.00)$ & $74.90(47.00-126.00)$ \\
\hline Diuresis mL/kg/h (RI) & $0.00(0.00-0.60)^{\star}$ & $0.70(0.00-1.50)^{*}$ \\
\hline Balance de líquidos $\mathrm{mL}(\mathrm{RI})$ & $3,400(2,200-4,600)$ & $2,500(1,500-4,250)$ \\
\hline $\mathrm{pH}(\mathrm{RI})$ & $7.21(7.10-7.33)^{*}$ & $7.39(7.35-7.41)^{*}$ \\
\hline $\mathrm{PaCO}_{2} \mathrm{mmHg}(\mathrm{RI})$ & $35(28-41)$ & $35(30-39)$ \\
\hline $\mathrm{HCO}_{3}^{-} \mathrm{mEq} / \mathrm{L}(\mathrm{DE})$ & $14.86( \pm 5.24)^{*}$ & $21.26( \pm 4.12)^{*}$ \\
\hline Base mEq/L (RI) & $-13.70(-21.00-7.80)^{*}$ & $-3.50(-6.20-1.00)^{*}$ \\
\hline Lactato mEq/L (RI) & $3.80(2.20-6.80)^{*}$ & $2.30(1.70-3.30)^{\star}$ \\
\hline DIFa mEq/L (RI) & $-4.00(-7.00-0.00)$ & $-2.00(-6.00-0.00)$ \\
\hline DIFe mEq/L (RI) & $9.80(5.50-9.93)$ & $6.75(4.50-9.83)$ \\
\hline GIF mEq/L (RI) & $-11.83(-15.00-7.85)^{\star}$ & $-9.93(-12.80-4.88)^{\star}$ \\
\hline Ventilación mecánica, días (RI) & $3(1-5)^{\star}$ & $4(3-6)^{*}$ \\
\hline Estancia de UCI, días (RI) & $3(2-6)^{*}$ & $5(4-8)^{*}$ \\
\hline TRRLC (\%) & 45.2 & 57.3 \\
\hline Mortalidad (\%) & $65.1^{*}$ & $36.0^{*}$ \\
\hline
\end{tabular}

Abreviaturas: $\mathrm{RI}=$ rango intercuartilar; $\mathrm{DE}=$ desviación estándar; $\mathrm{cm}=$ centímetros; $\mathrm{kg}=$ kilogramos; SAPS II = Simplified Acute Physiology Score II; $\mathrm{SvCO}_{2}=$ saturación venosa central de oxígeno; $\Delta \mathrm{p}(\mathrm{v}-\mathrm{a}) \mathrm{CO}_{2}=$ delta de presión venoarterial de dióxido de carbono; $\mathrm{mmHg} / \mathrm{mL}$ = milímetros de mercurio/mililitro; $\mu \mathrm{g} / \mathrm{kg} / \mathrm{min}=$ microgramos $/ \mathrm{kilogramo} /$ minuto; $\mathrm{mg} / \mathrm{dL}=$ miligramos $/$ decilitros; $\mathrm{mL} / \mathrm{kg} / \mathrm{h}=$ mililitros $/$ kilogramo/hora; $\mathrm{mEq} / \mathrm{L}=$ miliequivalentes/litro; $\mathrm{pH}=$ potencial de hidrogeniones; $\mathrm{PaCO}_{2}=$ presión arterial de dióxido de carbono; $\mathrm{mmHg}=$ milímetros de mercurio; $\mathrm{HCO}_{3}=$ bicarbonato; $\mathrm{mEq} / \mathrm{L}=$ miliequivalentes/itro; $\mathrm{DIFa}=$ diferencia de iones fuertes aparente; $\mathrm{DIFe}=$ diferencia de iones fuertes efectiva; GIF = gap de iones fuertes; $\mathrm{UCI}=$ Unidad de Cuidados Intensivos; TRRLC = terapia de reemplazo renal lenta continua; ${ }^{*}=p<0.05$.
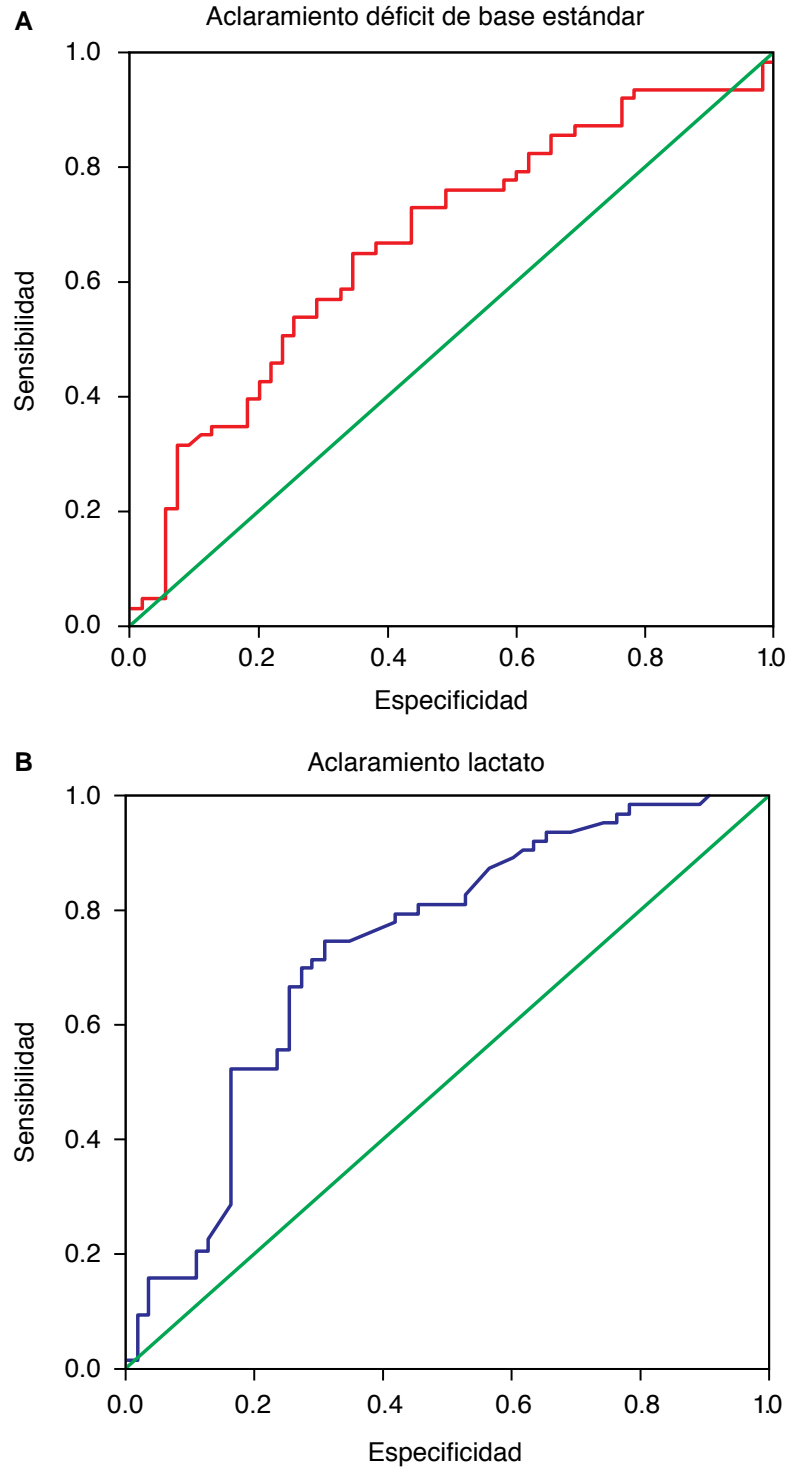

Figura 1: Curva ROC del porcentaje (\%) de aclaramiento del déficit de base estándar y del lactato para mortalidad. Abreviatura: $\mathrm{ROC}=$ Receiver Operating Characteristics.

do de carbono) o por problemas respiratorios, ${ }^{23}$ aunque en el contexto clínico probablemente no existe diferencia entre utilizar uno $u$ otro. ${ }^{24}$ Conocemos bien las implicaciones relacionadas con la morbimortalidad dependiendo de la severidad de la acidosis metabólica, pero el problema subyacente siempre será más importante. ${ }^{25}$ Entonces, el aclaramiento del déficit de base estándar se traduce en mejoría de la acidosis metabólica a través del tiempo y probablemente sea un reflejo de la recuperación del problema infeccioso. De hecho, estudios han documentado que la variación (incremento) del déficit de base estándar en pacientes con choque séptico es un factor de riesgo independiente para la mortalidad (OR 5.68). ${ }^{26}$

En nuestro estudio los pacientes que tuvieron $<11 \%$ de aclaramiento de déficit de base estándar presentaron 
mayor mortalidad respecto aquellos con aclaramiento $\geq$ $11 \%$ con 65.1 y $36 \%(p<0.05)$ respectivamente. Otras variables, que reflejan el grado de acidosis metabólica como son el $\mathrm{pH}, \mathrm{HCO}_{3}^{-}$, lactato y $\mathrm{GIF}$, tuvieron diferencia estadísticamente significativa entre ambos grupos, mostrando persistencia o mayor severidad de la acidosis metabólica a las 24 horas en los pacientes con aclaramiento $<11 \%$. Lo anterior muestra que el estado permanente de acidosis metabólica influye de manera negativa en los resultados. Hoy sabemos que la acidosis metabólica secundaria a lactato y aniones no medidos está asociada con mortalidad a diferencia de otras causas. ${ }^{27}$ La persistencia de la acidosis metabólica en el contexto de choque séptico será el reflejo del progreso de la causa subyacente, o bien la ausencia en la resolución de la misma sencillamente tiene un carácter secundario.

Según el modelo de Stewart la acidosis metabólica es consecuencia de alteraciones en la DIFa (diferencia de iones fuertes aparente) o hipercloremia e incremento del agua libre y de las alteraciones en la DIFe (diferencia de iones fuertes efectiva) o hiperfosfatemia e hiperalbuminemia. Agregado a estas cuatro razones (cloro, agua, fosforo y albumina) debemos sumar dos razones más: lactato y aniones no médi$\cos ^{28,29}$ Estudios recientes han documentado que la causa más frecuente de acidosis metabólica en los pacientes críticamente enfermos son los aniones no medidos, pero la de mayor mortalidad es la acidosis láctica. ${ }^{27}$ Por lo que nuestro interés debería estar centrado en el aclaramiento o disminución de estas dos variables.

Se ha documentado que el aclaramiento de lactato $<19 \%$ en 24 horas en pacientes críticamente enfermos incrementa el riesgo de muerte (OR 4.11, IC 95\% 3.23$5.21, p=0.001),{ }^{30}$ misma situación que ocurre con los aniones no medidos, los cuales son factor de riesgo independiente de mortalidad en los pacientes con choque séptico (OR 2.5, IC 95\% 1.03-6.2, $p=0.0001$ ). ${ }^{31}$ Consideramos que la ausencia de diferencia estadística en la DIFa y la DIFe a las 24 horas, es el reflejo de la poca o nula influencia sobre la mortalidad de la acidosis metabólica secundaria a hipercloremia, hiperfosfatemia, hiperalbuminemia y al exceso de agua libre. Pues, como lo mencionamos previamente, la acidosis metabólica se convierte en un factor de riesgo independiente para mor- talidad cuando es secundaria a lactato o aniones no medidos. ${ }^{32}$ Entonces, las alteraciones ácido-base se presentan prácticamente en todos los pacientes ingresados
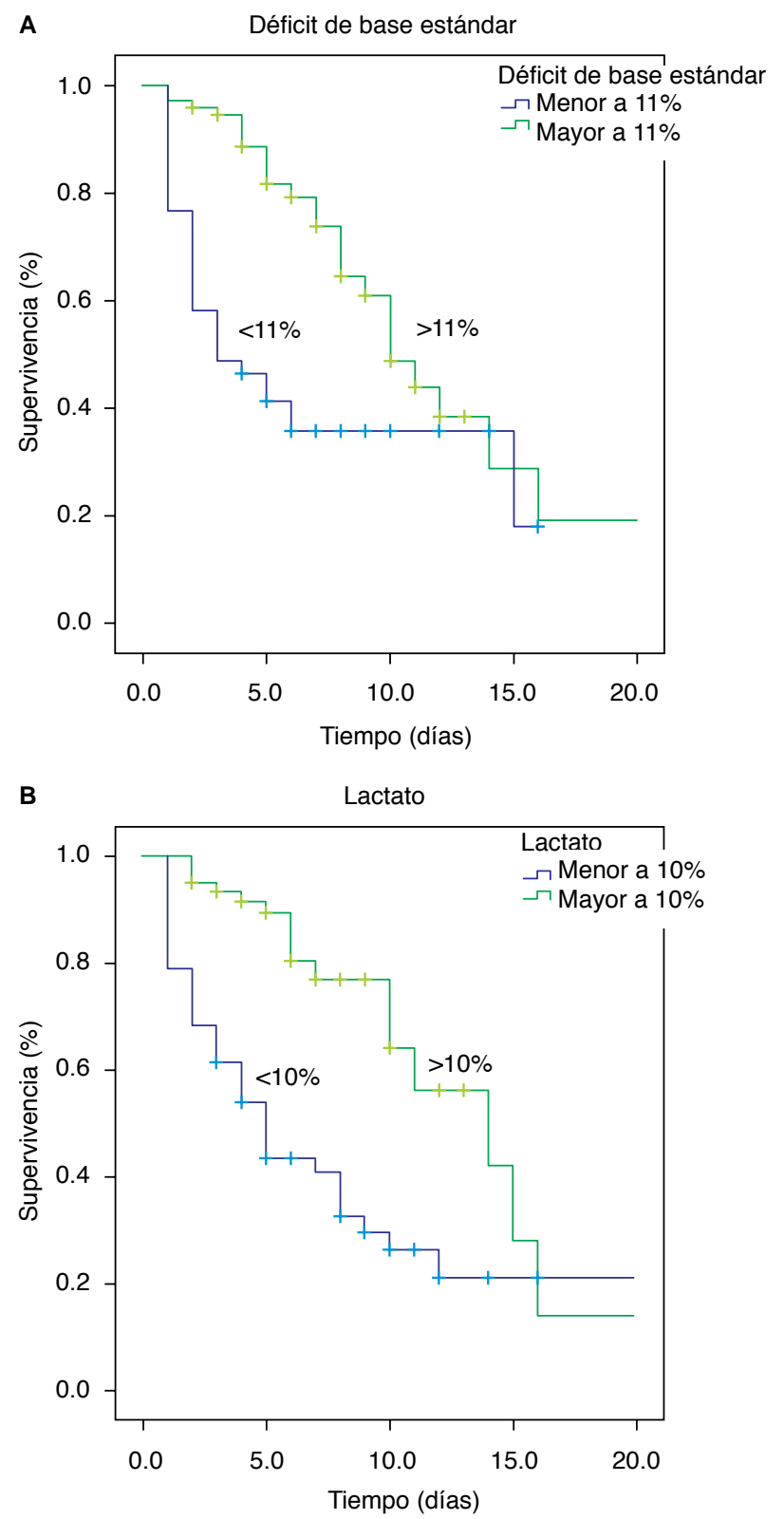

Figura 2: Análisis de supervivencia Kaplan-Meier para el porcentaje (\%) de aclaramiento del déficit de base estándar y de lactato. Abreviatura: Log Rank $p<0.05$.

Tabla 2: Análisis de la curva ROC del porcentaje (\%) de aclaramiento del déficit de base estándar y del lactato para mortalidad.

\begin{tabular}{|c|c|c|c|c|c|c|c|}
\hline Variable & $\mathrm{ABC}$ & $\mathrm{p}$ & \multicolumn{2}{|c|}{ IC $95 \%$} & Punto de corte & $\mathrm{S}$ & $E$ \\
\hline Aclaramiento del déficit de base estándar (\%) & 0.68 & 0.00 & 0.57 & 0.77 & 11 & 72 & 51 \\
\hline
\end{tabular}

Abreviaturas: $A B C=$ Área bajo la curva. IC = Intervalo de confianza. $S=$ Sencibilidad. $E=$ Especificidad. Se comparararon las ABC mediante la prueba de Hanley McNeil sin encontarar diferencia significativa $(p=\geq 0.05)$ en el desempeño de los puntos de corte seleccionados para mortalidad. 
a la $\mathrm{UCl}$, de hecho, pueden ser la principal razón de su ingreso. Generalmente tienen un origen secundario pues ocurren como una complicación de enfermedad aguda, además, por un lado, resultan útiles para evaluar el pronóstico de los pacientes críticamente enfermos desde su ingreso y según su evolución a través del tiempo, ${ }^{33,34}$ y por otro lado, la edad per se es un factor de riesgo independiente de la mortalidad en choque séptico ${ }^{35} \mathrm{y}$ la severidad de la enfermedad es determinante para la supervivencia. Las diferentes escalas pronósticas tienen un incremento lineal conforme la edad aumenta, ${ }^{36}$ por lo que no resulta nueva la diferencia estadística que presentan la edad (65 vs 63) y el SAPS II (72.79 vs 60.21) entre el grupo de aclaramiento de déficit de base estándar $<11 \%$ y $\geq 11 \%$ respectivamente.

Finalmente, no queda en duda la utilidad del lactato para predecir mortalidad en los pacientes con choque séptico, pero al realizar la comparación del $A B C$ mediante la prueba de Hanley McNeil no se encontró diferencia significativa $(p>0.05)$ en el desempeño de los puntos de corte seleccionados para mortalidad entre el porcentaje de aclaramiento del déficit de base estándar y del porcentaje del lactato, lo que nos indica que su capacidad pronóstica es similar, pues se puede utilizar el déficit de base estándar en ausencia del lactato, ya que en nuestro medio no es infrecuente no tenerlo disponible.

\section{CONCLUSIONES}

El aclaramiento del déficit de base estándar $<11 \%$ a las 24 horas de ingreso a la Unidad de Cuidados Intensivos incrementa el riesgo de muerte en el choque séptico. El estado permanente de acidosis metabólica influye de manera negativa en los resultados, principalmente cuando es secundario a lactato o aniones no medidos. El porcentaje de aclaramiento del lactato en choque séptico es una variable ampliamente estudiada, pero el porcentaje de aclaramiento del déficit de base estándar es una excelente alternativa, pues su desempeño para predecir mortalidad tiene una capacidad pronóstica similar.

\section{BIBLIOGRAFÍA}

1. Martin GS, Mannino DM, Moss M. The effect of age on the development and outcome of adult sepsis. Crit Care Med. 2006;34:15-21.

2. Prescott $\mathrm{HC}$, Angus DC. Enhancing recovery from sepsis: a review. JAMA. 2018;319(1):62-75.

3. Carrillo R, Carrillo JR, Carrillo LD. Estudio epidemiológico de la sepsis en unidades de terapia intensiva mexicanas. Cir Ciruj. 2009;77:301-308.

4. Gorordo-Delsol LA. Sepsis: el enemigo oculto entre líneas. Rev Med Inst Mex Seg Soc. 2017;55(4):423.

5. Vincent JL, De Backer D. Circulatory shock. $N$ Engl $J$ Med. 2013;369(18):1726-1734.

6. Angus DC, van der Poll T. Severe sepsis and septic shock. $N$ Engl J Med. 2013;369(9):840-851.
7. Kofstad J. Base excess: a historical review-has de calculation of base excess been more standardized the last 20 years? Clin Chim Acta. 2001;307(1-2):193-195.

8. Severinhaus JW. Siggaard-Andersen and the great transatlantic acid base debate. Scand J Clin Lab Invest. 1993;53:99-104.

9. Häubi SC, Moreno-Santillán A, de León-Ponce MD, BrionesVega CG, Meneses-Calderón J, Orenday-Aréchiga ME, et al. Teoría ácido-básica de Stewart, un nuevo paradigma en medicina crítica. Rev Mex Anest. 2006;29(4):240-244.

10. Scheiner B, Lindner G, Reiberger T, Schneeweiss B, Trauner M, Zauner $\mathrm{C}$, et al. Acid-base disorders in liver disease. J Hepatol. 2017;67(5):1062-1073.

11. Sánchez-Díaz JS, Martínez-Rodríguez EA, Méndez-Rubio LP, Peniche-Moguel KG, Huanca-Pacaje JM, López-Guzmán C, et al. Equilibrio ácido-base. Puesta al día. Teoría de HendersonHasselbalch. Med Int Mex. 2016;32:646-660.

12. Knaus WA, Draper EA, Wagner DP, Zimmerman JE. APACHE II: a severity of disease classification system. Crit Care Med. $1985 ; 13(10): 818-829$.

13. Hajjar LA, Nakamura RE, de Almeida JP, Fukushima JT, Hoff $\mathrm{PM}$, Vincent JL, et al. Lactate and base deficit are predictors of mortality in critically ill patients with cancer. Clinics. 2011;66(12):2037-2042.

14. Vincent JL. Serial blood lactate levels reflect both lactate production and clearance. Crit Care Med. 2015;43:e209.

15. Vincent JL, Dufaye P, Berré J, Leeman M, Degaute JP, Kahn RJ. Serial lactate determinations during circulatory shock. Crit Care Med. 1983;11(6):449-451.

16. Puskarich MA, Jones AE. Clearing lactate is clearly better ... but how much? Crit Care Med. 2014;42:2149-2150.

17. Vincent JL, Quintairos E Silva A, Couto L Jr, Taccone FS. The value of blood lactate kinetics in critically ill patients: a systematic review. Crit Care. 2016;20(1):257.

18. Nichol A, Bailey M, Egi M, Pettila V, French C, Stachowski E, et al. Dynamic lactate indices as predictors of outcome in critically ill patients. Crit Care. 2011;15:R242.

19. Haas SA, Lange T, Saugel B, Petzoldt M, Fuhrmann V, Metschke $M$, et al. Severe hyperlactatemia, lactate clearance and mortality in unselected critically ill patients. Intensive Care Med. 2016;42:202-210.

20. Zante B, Reichenspurner H, Kubik M, Kluge S, Schefold JC, Pfortmueller CA. Base excess is superior to lactate-levels in prediction of ICU mortality after cardiac surgery. PLoS One. 2018;13(10):e0205309.

21. Husain FA, Martin MJ, Mullenix PS, Steele SR, Elliott DC. Serum lactate and base deficit as predictors of mortality and morbidity. Am J Surg. 2003;185(5):485-491.

22. Montassier E, Batard E, Segard J, Hardouin JB, Martinage A, Le Conte $\mathrm{P}$, et al. Base excess is an accurate predictor of elevated lactate in ED septic patients. Am J Emerg Med. 2012;30(1):184-187.

23. Gunnerson KJ, Saul M, He S, Kellum JA. Lactate versus nonlactate metabolic acidosis: a retrospective outcome evaluation of critically ill patients. Critical Care. 2006;10:R22.

24. FitzSullivan E, Salim A, Demetriades D, Asensio J, Martin MJ. Serum bicarbonate may replace the arterial base deficit in the trauma intensive care unit. Am J Surg. 2005;190(6):941946.

25. Al-Jaghbeer M, Kellum JA. Acid-base disturbances in intensive care patients: etiology, pathophysiology and treatment. Nephrol Dial Transplant. 2015;30(7):1104-1111.

26. Palma LC, Ferreira GF, Amaral A, Brauer L, Azevedo LCP, Park $M$. Acidosis and mortality in severe sepsis and septic shock evaluated by base excess variation. Crit Care. 2003;7(Suppl 3):P39.

27. Masevicius FD, Rubatto Birri PN, Risso Vazquez A, Zechner FE, Motta MF, Valenzuela Espinoza ED, et al. Relationship of at admission lactate, unmeasured anions, and chloride to the outcome of critically ill patients. Crit Care Med. 2017;45(12):e1233-e1239.

28. Sánchez DJS, Monares ZE, Meneses OC, Rodríguez MEA, García MRC, Peniche MKG, et al. Soluciones balanceadas: cloro el «nuevo villano». Rev Asoc Mex Med Crit y Ter Int. 2017;31(3):152-158. 
29. Maciel AT, Park M. Unmeasured anions account for most of the metabolic acidosis in patients with hyperlactatemia. Clinics (Sao Paulo). 2007;62(1):55-62.

30. Masyuk M, Wernly B, Lichtenauer M, Franz M, Kabisch B, Muessig JM, et al. Prognostic relevance of serum lactate kinetics in critically ill patients. Intensive Care Med. 2019;45(1):55-61.

31. Pin GE, Sánchez DJS, Martínez REA, García MRC, Peniche MKG, Calyeca SMV. Clasificación del choque séptico a partir de los iones no medidos. Rev Asoc Mex Med Crit y Ter Int. 2018;32(1):13-19.

32. Antonini B, Piva S, Paltenghi M, Candiani A, Latronico N. The early phase of critical illness is a progressive acidic state due to unmeasured anions. Eur J Anaesthesiol. 2008;25(7):566-571.

33. Corey HE. Stewart and beyond: new models of acid-base balance. Kidney Int. 2003;64(3):777-87.

34. Maciel AT, Park M. Differences in acid-base behavior between intensive care unit survivors and nonsurvivors using both a physicochemical and a standard base excess approach: a prospective, observational study. J Crit Care. 2009;24(4):477-483.

35. Lemay AC, Anzueto A, Restrepo MI, Mortensen EM. Predictors of long-term mortality after severe sepsis in the elderly. $A m ~ J$ Med Sci. 2014;347(4):282-288.
36. Mayer-Oakes SA, Oye RK, Leake B. Predictors of mortality in older patients following medical intensive care: the importance of functional status. J Am Geriatr Soc. 1991;39:862-868.

Patrocinios. Relación de conflictos de interés: el presente trabajo no recibió patrocinios, sólo se utilizaron recursos propios de nuestro hospital. Los autores declaramos no tener conflictos de interés.

Correspondencia:

Dr. Jesús Salvador Sánchez Díaz

Av. Cuauhtémoc S/N, Formando Hogar, 91897, Veracruz, Veracruz.

Teléfono: 2299342031

E-mail: drsalvadorsanchezdiaz@gmail.com 